Article

\title{
Dual-Tunable Broadband Terahertz Absorber Based on a Hybrid Graphene-Dirac Semimetal Structure
}

\author{
Jiali Wu, Xueguang Yuan $\left(\mathbb{D}\right.$, Yangan Zhang *, Xin Yan ${ }^{(1)}$ and Xia Zhang \\ State Key Laboratory of Information Photonics and Optical Communications, \\ Beijing University of Posts and Telecommunications, Beijing 100876, China; Flowing_Kinga@bupt.edu.cn (J.W.); \\ yuanxg@bupt.edu.cn (X.Y.); xyan@bupt.edu.cn (X.Y.); xzhang@bupt.edu.cn (X.Z.) \\ * Correspondence: zhang@bupt.edu.cn; Tel.: +86-10-61198040
}

Received: 30 October 2020; Accepted: 9 December 2020; Published: 11 December 2020

check for updates

\begin{abstract}
A dual-controlled tunable broadband terahertz absorber based on a hybrid graphene-Dirac semimetal structure is designed and studied. Owing to the flexible tunability of the surface conductivity of graphene and relative permittivity of Dirac semimetal, the absorption bandwidth can be tuned independently or jointly by shifting the Fermi energy through chemical doping or applying gate voltage. Under normal incidence, the device exhibits a high absorption larger than $90 \%$ over a broad range of 4.06-10.7 THz for both TE and TM polarizations. Moreover, the absorber is insensitive to incident angles, yielding a high absorption over $90 \%$ at a large incident angle of $60^{\circ}$ and $70^{\circ}$ for $\mathrm{TE}$ and TM modes, respectively. The structure shows great potential in miniaturized ultra-broadband terahertz absorbers and related applications.
\end{abstract}

Keywords: absorber; graphene; Dirac semimetal; dual-controlled; broadband

\section{Introduction}

Terahertz (THz) absorbers have been widely investigated due to their wide applications in electromagnetic radiation, radiation, sensation, and thermal imaging [1-3]. A conventional terahertz absorber unit cell is typically composed of metallic materials, which can only operate at fixed predesigned frequencies with a relatively narrow absorption bandwidth [4,5]. In recent years, graphene and 3D bulk Dirac semimetal (BDS) are emerging as promising candidates for dynamically tunable broadband absorbers in the terahertz range [6]. Graphene, a new type of two-dimensional material, has attracted great interest due to its remarkable optical properties, high carrier mobility, and tunable surface conductivity within the terahertz frequency range [7]. Similarly, the relative permittivity of BDS, a material that can be considered as "3D graphene", can also be dynamically controlled by an external gate voltage [8,9]. Up to now, some graphene- or Dirac-semimetal-based devices have been reported in the terahertz range [10-18]. Nevertheless, the bandwidth of these absorbers is not wide enough, and the tuning method is relatively monotonous. Therefore, it is worthwhile to develop advanced $\mathrm{THz}$ absorbers with much higher bandwidth and more flexible tuning ways.

In this work, a dual-controlled ultra-broadband $\mathrm{THz}$ absorber based on a hybrid graphene-Dirac semimetal multilayer structure is proposed and studied. By simply shifting the Fermi levels of graphene and Dirac semimetal, the proposed structure shows tunable absorption over a broadband wavelength range. The simulated results show that under normal incidence, the device exhibits a high absorption larger than $90 \%$ over a broad range of $4.06-10.7 \mathrm{THz}$ for both TE and TM polarizations. Moreover, the absorber is insensitive to incident angles, yielding a high absorption over $90 \%$ at a large incident angle of $60^{\circ}$ and $70^{\circ}$ for TE and TM modes, respectively. The physical mechanisms are also elucidated by the impedance matching theory and electric field analyses. 


\section{Materials and Design}

\subsection{Graphene Material}

It is known that graphene is a potential material to design reconfigurable $\mathrm{THz}$ devices due to the tunable surface conductivity $\sigma_{g}(\omega)$, which can be indicated by the Kubo's formula [19]:

$$
\sigma_{g}(\omega)=\frac{e^{2} k_{B} T}{\pi h^{2}}\left(\frac{\mu}{k_{B} T}+2 \ln \left(e^{-\frac{\mu}{k_{B} T}}+1\right)\right) \frac{-i}{\omega-i \tau^{-1}}+\frac{-i e^{2}}{4 \pi h} \ln \left(\frac{2|\mu|-\left(\omega-i \tau^{-1}\right) h}{2|\mu|+\left(\omega-i \tau^{-1}\right) h}\right)
$$

where $\mu$ is the Fermi energy (or chemical potential) of graphene which can be controlled by applying bias voltage or chemical doping, $T$ is the temperature, $i$ is the imaginary unit, $e$ is the charge number of an electron, $k_{B}$ is Boltzmann's constant, $h=h / 2 \pi$ is the reduced Plank's constant, and $\tau$ is the relaxation time. In the following simulations, we set the parameters as $T=293 \mathrm{~K}$ and $\tau=0.1$ ps. From Equation (1), we can learn that the conductivity $\sigma_{g}(\omega)$ is related to frequency $\omega$ and Fermi energy $\mu$ adjusted by gate voltage. Furthermore, the surface impedance of graphene $Z_{g}$ is expressed as $Z_{g}=1 / \sigma_{g}, R_{g}=\operatorname{Re}\left(Z_{g}\right)$, and $X_{g}=\operatorname{Im}\left(Z_{g}\right)$, where $R_{g}$ is the resistance of graphene and $X_{g}$ is reactance of graphene. The frequency-dependent resistance $R_{g}$ and reactance $X_{g}$ are shown in Figure $1 a$. It can be found that at a fixed Fermi energy, the resistance $R_{g}$ keeps almost constant, while reactance $X_{\mathrm{g}}$ increases linearly with increasing frequency.

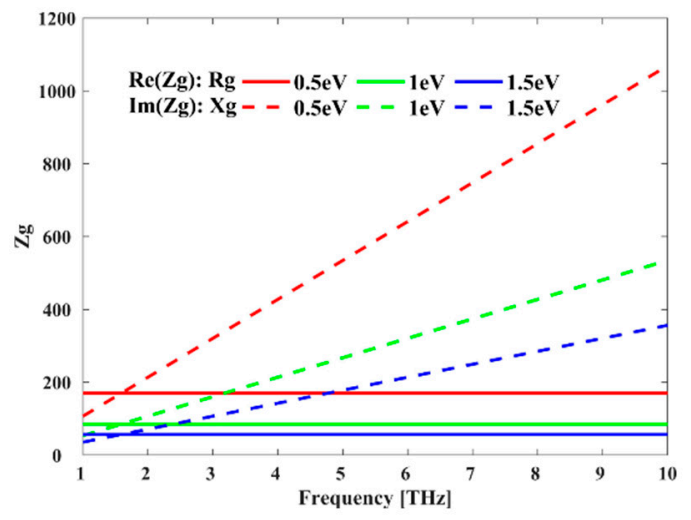

(a)

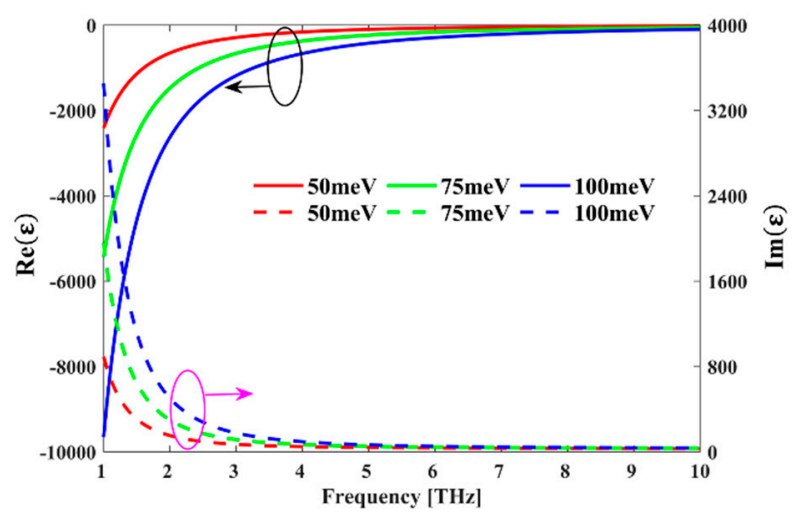

(b)

Figure 1. (a,b) Frequency-dependent surface impedance Zg of graphene and permittivity of bulk Dirac semimetal (BDS) at different Fermi energies, respectively.

\subsection{BDS Material}

In the $\mathrm{THz}$ range, the relative permittivity of BDS can be expressed as [20]:

$$
\varepsilon=\varepsilon_{b}+i \frac{\sigma}{\varepsilon_{0} \omega}
$$

where $\varepsilon_{0}$ is the permittivity of vacuum and $\varepsilon_{b}=1$.

The dynamic conductivity $\sigma$ can be written as [21]:

$$
\begin{gathered}
\operatorname{Re} \sigma=\frac{e^{2} g k_{F}}{24 \pi h}\left(\frac{h \omega}{E_{F}}+\frac{i h \tau^{-1}}{E_{F}}\right) \theta\left(\frac{h \omega}{E_{F}}+\frac{i h \tau^{-1}}{E_{F}}-2\right) \\
\operatorname{Im} \sigma=\frac{e^{2} g k_{F}}{24 \pi^{2} h}\left[\frac{4}{\frac{h \omega}{E_{F}}+\frac{i h \tau^{-1}}{E_{F}}}-\frac{h \omega}{E_{F}}+\frac{i h \tau^{-1}}{E_{F}} \ln \left(\frac{4 \varepsilon_{c}^{2}}{\left|\left(\frac{h \omega}{E_{F}}+\frac{i h \tau^{-1}}{E_{F}}\right)^{2}-4\right|}\right)\right]
\end{gathered}
$$


where $h$ is the reduced Plank's constant, $k_{F}=E_{F} / h v_{F}$ is the Fermi momentum, $E_{F}$ is the Fermi energy applied to the BDS, $v_{F}=10^{6} \mathrm{~ms}^{-1}$ is the Fermi velocity, $\tau=4.5 \times 10^{-13}, g=40, \varepsilon_{c}=3$, and $\theta(t)$ is the Riemann-Siegel theta function $\theta(t)$, which can be illustrated as [22]:

$$
\theta(t)=-\frac{i}{2}\left[\ln \Gamma\left(\frac{1}{4}+\frac{i t}{2}\right)-\ln \Gamma\left(\frac{1}{4}-\frac{i t}{2}\right)\right]-\frac{t \ln (\pi)}{2}
$$

From Equations (2)-(5), we can see that the permittivity of BDS can also be controlled by Fermi energy. Figure $1 \mathrm{~b}$ displays the frequency-dependent real and imaginary parts of BDS at different Fermi energies. It reveals that the real and imaginary parts of permittivity vary quickly in the range of 1-5 THz. The resonance frequency is mainly influenced by the real part of permittivity, while the loss is affected by the imaginary parts.

\subsection{Design Method}

The schematic diagram of the dual-controlled ultra-broadband $\mathrm{THz}$ absorber is illustrated in Figure 2. The structure is composed of five parts, which are top monolayer graphene pattern film, upper $\mathrm{Al}_{2} \mathrm{O}_{3}$ layer with a relative permittivity of 2.28 and loss tangent of 0.04 , BDS complementary pattern layer, lower $\mathrm{Al}_{2} \mathrm{O}_{3}$ layer, and gold layer with conductivity $\sigma=4.56 \times 10^{7} \mathrm{~S} / \mathrm{m}$ from top to bottom. In this work, AlCuFe is used as the BDS material [16].

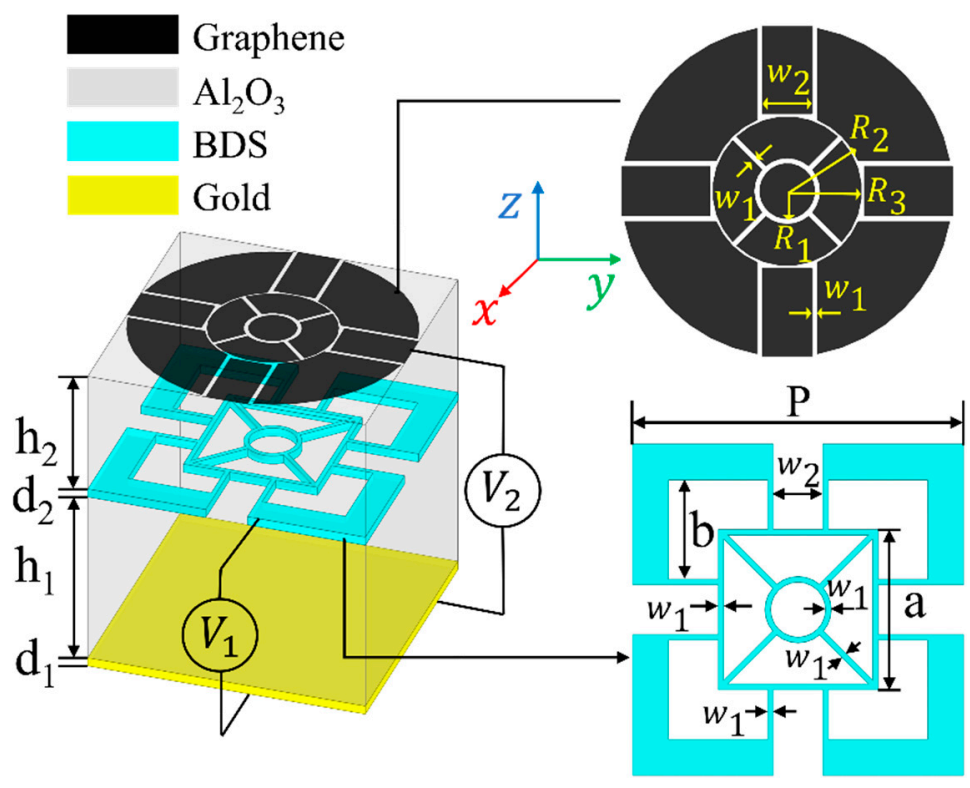

Figure 2. Schematic diagram of the proposed $\mathrm{THz}$ absorber.

The top graphene patterned layer is to match the impedance of free space at a specific working frequency band, so as to achieve no reflection of the incident wave. The BDS pattern of the middle layer is to expand the absorption bandwidth. The basic function of the $\mathrm{Al}_{2} \mathrm{O}_{3}$ layer is to provide resonance absorption space, and the bottom gold layer is used to reflect the incident wave. In order to satisfy the polarization and incident angle insensitivity, we have designed a symmetrical structure as shown in Figure 2.

The reasons for designing this structure are as follows: (1) In the patterned graphene structure, due to the localized surface plasmon resonance, most of the electric field will be limited to the edge of the annular graphene. This resonance can effectively capture light energy to enhance the absorption rate. More circular channels in the graphene pattern can be arranged to limit the electric field distribution on the edge of the graphene, thereby increasing the absorption rate. Therefore, in our proposed structure, we use $w_{1}, w_{2}, R_{1}, R_{2}$, and $R_{3}$ to construct circular rings and square channels in the graphene pattern. 
(2) In the design of the patterned BDS structure, on the one hand, the method of designing multiple small channels similar to the graphene layer is continued to be used; on the other hand, the structure is supposed to complement to the upper graphene pattern. In this case, the two absorption layers would resonate at different frequencies, thus extending the absorption bandwidth of the structure. In our proposed structure, the BDS pattern is controlled by $\mathrm{w}_{1}, \mathrm{w}_{2}, \mathrm{a}$, and $\mathrm{b}$. (3) In the conventional absorbing material model, in order to ensure that the wavelength in the dielectric layers forms a stable standing wave of electromagnetic waves, the thickness of the dielectric layer satisfies $h=(2 n+1) \lambda / 4$, where $n=0,1,2 \ldots$. In that case, the incident electromagnetic wave would interfere destructively with the reflected wave from the bottom metal layer, leading to the absorption of the electromagnetic wave energy by the structure. But for the metamaterial absorber, the thickness of the dielectric layer does not need to be a quarter wavelength as the dielectric constant and permeability of the metamaterial are controllable, which facilitates the realization of the absorber structure. In our work, the size of the two dielectric layers of graphene-BDS hybrid absorber is $4 \mu \mathrm{m}$ and $2.8 \mu \mathrm{m}$, which are obtained from scanning optimization in the simulation software in consideration of the requirements of structural miniaturization and high absorption performance.

Ansys HFSS is used for the simulation of the proposed structure. The THz wave impinges on the graphene pattern from the air. Periodic linked boundary conditions (primary and secondary) are adopted in the $\mathrm{x}$ - and $\mathrm{y}$-directions and Floquet port excitation in the $\mathrm{z}$-direction. To better describe a graphene film, the graphene layer is considered as a two-dimensional conductive surface with the impedance boundary of resistance and reactance in the software simulations. For BDS material, the relative permittivity is created by importing relevant data obtained from Equations (2)-(5). Since the thickness of the bottom gold film is considerably larger than the skin depth, the electromagnetic waves can hardly be transmitted through the bottom metal plate, leading to $S_{21}=0$ and absorbance $\mathrm{A}=1-\left|S_{11}\right|^{2}$. The detailed dimensional parameters of the proposed absorber are listed in Table 1 . These specific parameter values are generated by setting optimization goals that express as 1-(mag(S(FloquetPort1:1,FloquetPort1:1) $))^{\wedge} 2>0.9$ in Ansys HFSS.

Table 1. Detailed dimensional parameters of the proposed absorber structure.

\begin{tabular}{cccccc}
\hline Parameter & Size $(\mu \mathrm{m})$ & Parameter & Size $(\mu \mathrm{m})$ & Parameter & Size $(\mu \mathrm{m})$ \\
\hline $\boldsymbol{d}_{\mathbf{1}}$ & 0.2 & $P$ & 6 & $R_{2}$ & 1.4 \\
$\boldsymbol{d}_{\mathbf{2}}$ & 0.2 & $a$ & 2.9 & $R_{3}$ & $\left(\mathrm{a}-2 \times w_{1}\right) / 2$ \\
$\boldsymbol{h}_{\mathbf{1}}$ & 4 & $b$ & 1.8 & $w_{1}$ & 0.1 \\
$\boldsymbol{h}_{\mathbf{2}}$ & 2.8 & $R_{1}$ & 0.5 & $w_{2}$ & 0.9 \\
\hline
\end{tabular}

\section{Results and Discussions}

The absorption properties of different structures under normal incident TE and TM polarizations are shown in Figure 3. It can be seen that the BDS absorber exhibits a poor absorption lower than $90 \%$ over a broadband wavelength range of 1-13 THz. The graphene absorber shows high absorption above $90 \%$ from $4.72 \mathrm{THz}$ to $9.83 \mathrm{THz}$, yielding an effective absorption bandwidth of $5.11 \mathrm{THz}$. By combining graphene and BDS, the absorption bandwidth is significantly increased. At a Fermi energy of $1.5 \mathrm{eV}$ and $60 \mathrm{meV}$ for graphene and BDS, respectively, the hybrid graphene-BDS structure exhibits effective absorption (above $90 \%$ ) from $4.06 \mathrm{THz}$ to $10.7 \mathrm{THz}$, yielding a much broader absorption bandwidth of $6.64 \mathrm{THz}$. To the best of our knowledge, the metamaterial absorber with an absorption bandwidth higher than $6.6 \mathrm{THz}$ has not been reported yet. The center frequency $f_{c}$ is defined as $f_{c}=\left(f_{-}+f_{+}\right) / 2=7.38 \mathrm{THz}$, where $f_{-}$and $f_{+}$are the low- and high-frequency edges of $90 \%$ absorptance, respectively. Therefore, the fractional bandwidth (BW), the ratio of the absolute bandwidth to the center frequency is as high as $89.97 \%$. To further illustrate the performance of the hybrid absorber, main parameters of reported $\mathrm{THz}$ absorbers based on graphene or BDS are listed in Table 2 for comparisons. Compared with other structures, the hybrid absorber exhibits significantly 
larger bandwidth and higher fractional BW with a small number of layers, showing great potential in miniaturized broadband $\mathrm{THz}$ applications.

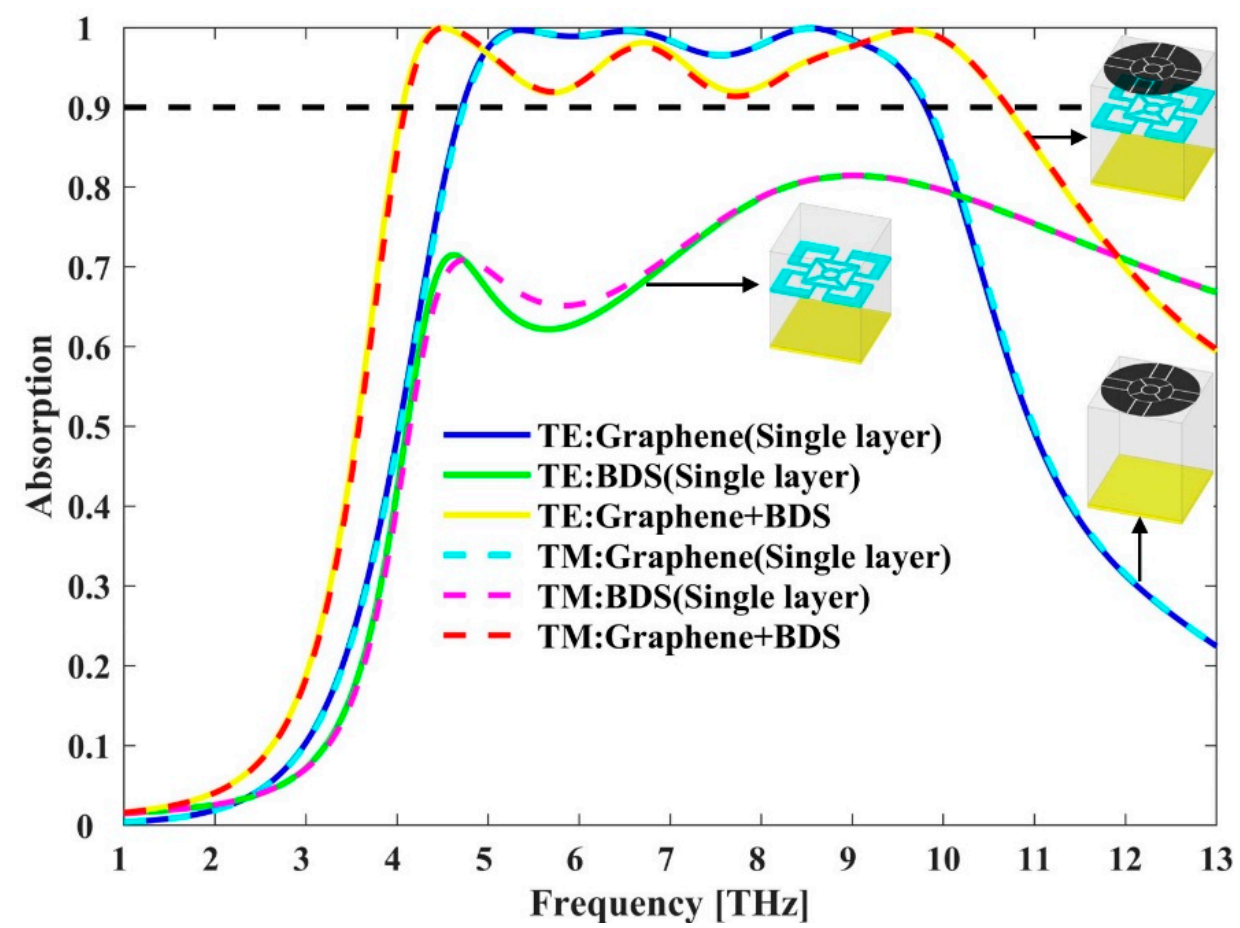

Figure 3. Absorption properties for different structures under normal incident TE and TM polarizations.

Table 2. Comparison of parameters of THz absorbers based on graphene or BDS.

\begin{tabular}{|c|c|c|c|c|c|c|}
\hline References & $\begin{array}{l}\text { Absorption } \\
\text { Band (THz) }\end{array}$ & $\begin{array}{c}\text { Fractional } \\
\text { BW }\end{array}$ & Layers & Tunable Material & Polarization-Insensitivity & Angle-Insensitivity \\
\hline [4] & $3-7.8$ & $88.8 \%$ & 8 & Graphene & insensitive & $50^{\circ}$ \\
\hline [23] & $5.50-9.10$ & $8.2 \%$ & 3 & Graphene & insensitive & $60^{\circ}$ \\
\hline [17] & $<0.1$ & $<5 \%$ & 1 & BDS & insensitive & $60^{\circ}$ \\
\hline [24] & $1.05-1.6$ & $42.5 \%$ & 2 & $\begin{array}{c}\text { Graphene and } \\
\text { vanadium dioxide }\end{array}$ & insensitive & $50^{\circ}$ \\
\hline This paper & $4.06-10.7$ & $89.97 \%$ & 2 & Graphene and BDS & insensitive & $60^{\circ}$ \\
\hline
\end{tabular}

Next, we studied the influence of graphene Fermi energy on absorption performance. Figure $4 a, b$ display the absorption spectra of the proposed hybrid structure as a function of frequency and Fermi energy $\mu$ under normal incidence TE and TM polarizations, respectively. The Fermi energy $E_{F}$ of BDS is fixed at $60 \mathrm{meV}$. When the Fermi level of graphene $\mu$ is 0 , only BDS functions as the absorption layer, and the effective absorption (above $90 \%$ absorption) is 0 . As $\mu$ increases, the absorption gradually increases, and multiple absorption peaks gradually appear, leading to a significantly broader bandwidth. It can be learned from Equation (1) and Figure 1a that the conductivity $\sigma_{g}(\omega)$ and surface impedance in graphene are related with Fermi energy $\mu$. As the Fermi level increases, the surface conductivity in graphene increases, and the plasma oscillation effect also increases, which ultimately leads to an increase in the absorption bandwidth. Therefore, the absorption performance of the hybrid structure can be effectively controlled by tuning the Fermi level of graphene in a broad wavelength range from 0 to $6.64 \mathrm{THz}$. 


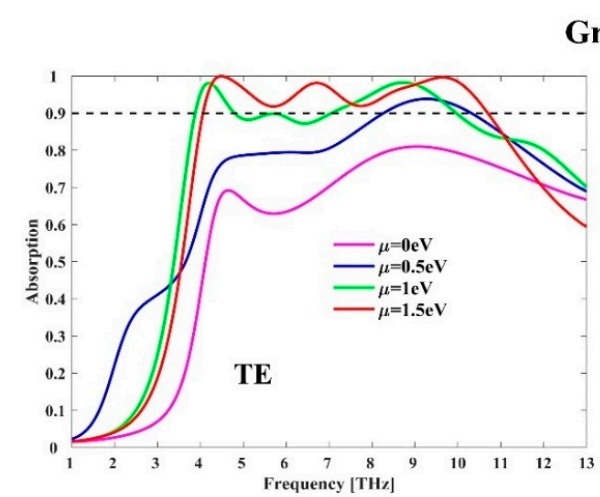

(a)

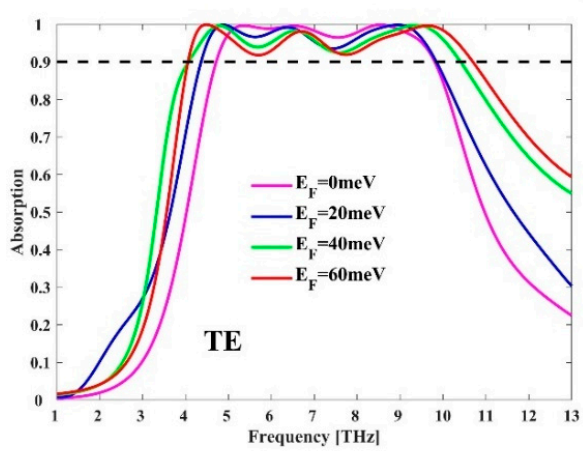

(c)

Graphene

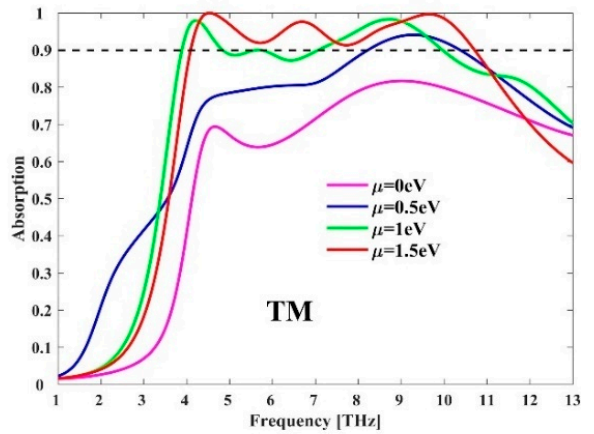

(b)

BDS

Figure 4. Absorption spectra with different graphene Fermi energy $\mathrm{u}=0 \mathrm{eV}, 0.5 \mathrm{eV}, 1 \mathrm{eV}$, and $1.5 \mathrm{eV}$ for (a) TE and (b) TM polarizations. Absorption spectra with different BDS Fermi energy $E_{F}=0 \mathrm{meV}$, $20 \mathrm{meV}, 40 \mathrm{meV}$, and $60 \mathrm{meV}$ for (c) TE and (d) TM polarizations.

BDS offers another possibility to tune the absorption performance of the hybrid structures. Figure $4 \mathrm{c}, \mathrm{d}$ present the absorption with different BDS Fermi energy $E_{F}$ of $0 \mathrm{meV}, 20 \mathrm{meV}, 40 \mathrm{meV}$, and $60 \mathrm{meV}$ under normal incidence for TE and TM polarizations, respectively. The graphene Fermi energy $\mu$ is fixed at $1.5 \mathrm{eV}$. With the increase of the BDS Fermi level, the first absorption peak exhibits a redshift while the second and third absorption peaks show a blueshift, resulting in an increasing absorption bandwidth. It can be explained as follows: in the low terahertz band, the resonance frequency is mainly influenced by the real part of permittivity. It can be learned from Figure $1 \mathrm{~b}$ that as the Fermi level increases, the real part of permittivity decreases, which leads to the decrease of resonance frequency and redshift of the absorption peak. In the high terahertz frequency band, the real part of BDS permittivity is close to 0 in Figure $1 b$. In this case, the resonance frequency is mainly affected by the localized plasmon resonance effect. As the Fermi level increases, the carrier density increases, leading to an increase of the plasmon resonance frequency and blueshift of the absorption peak. Thus, it can be concluded that the absorption performance of the hybrid absorber can be effectively regulated by means of tuning the resonance absorption peaks.

To sum up, owing to the tunability of absorption bandwidth by graphene Fermi energy and the control of resonance absorption peaks by BDS Fermi energy, our proposed absorber combines the advantages of being broadband-controlled and frequency-adjusted.

For $\mathrm{THz}$ absorbers, the sensitivity of polarization and incident angle is a significant issue in practical applications. Here, the absorption properties of the proposed structure under different polarization waves and incident angles are investigated, as shown in Figure 4. Here, the Fermi energy $\mu$ and EF of graphene and BDS are fixed at $1.5 \mathrm{eV}$ and $60 \mathrm{meV}$, respectively. As shown in Figure $5 \mathrm{a}, \mathrm{b}$, the absorbance is almost independent of the incident angle up to $50^{\circ}$ and $60^{\circ}$ for TE and TM modes, respectively. For TE, the absorptance remains over $80 \%$ when the incident angle is $\theta$ up 
to $60^{\circ}$. With increasing incident angle $\theta$, the absorptance gradually decreases and the absorption frequency is slightly expanded to a higher position. For TM polarized wave, the absorption is higher than $80 \%$ for incident angle up to $70^{\circ}$. However, the absorptance bandwidth slightly increases when $\theta$ becomes larger. Hence, the proposed absorber can tolerate a wide incident angle for both TE and TM polarizations. Figure $5 c$ depicts the absorption spectra under normal incidence with different polarization angles $\varphi$. It can be seen that the absorptance is insensitive to the polarization angle, which is attributed to the symmetry of the structure. Therefore, the proposed structure can be utilized as a polarization- and incident angle-insensitive broadband $\mathrm{THz}$ absorber.

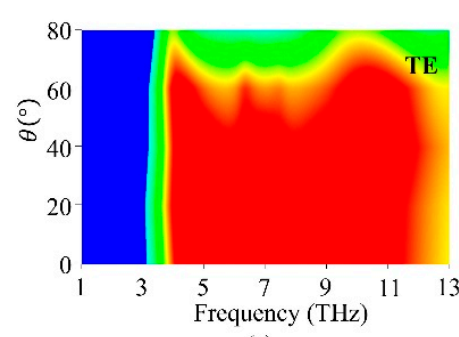

(a)

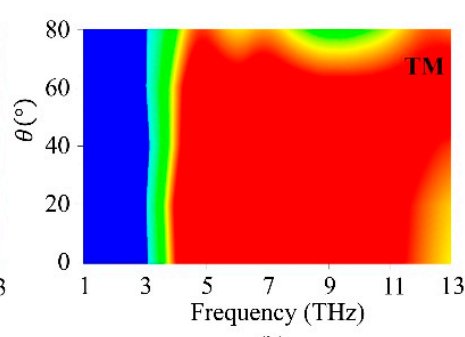

(b)

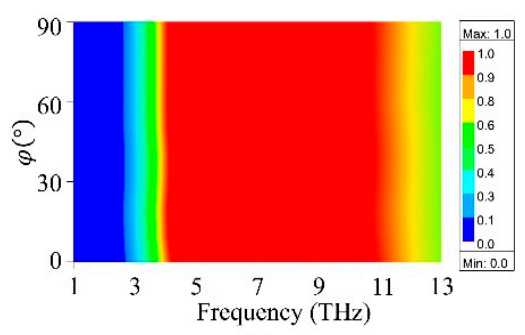

(c)

Figure 5. (a,b) Absorption maps as a function of incident angles $\theta$ for TE and TM polarization, respectively. (c) The absorption map for various polarization angles $\varphi$ under normal incidence.

To clarify the working principle, the absorption peaks located at 4.49,6.738, and $9.66 \mathrm{THz}$ are explained according to the impedance matching theory. The relative impedance ( $\mathrm{Zr}$ ) is calculated using Equations (6)-(10), where $d$ is the travelled distance and $k_{0}$ is the propagation constant of the wave at free space. The calculated values of the constitutive parameters for three absorption peaks are listed in Table 3. At all three frequencies, the real and imaginary parts of relative impedance $\mathrm{Zr}$ are very close to 1 and 0 , respectively. More generally, Figure 6 presents the real and imaginary parts of the relative impedance for TE polarized wave in full terahertz band. We can see that in the frequency range from $4.58 \mathrm{THz}$ to $10 \mathrm{THz}$, the real and imaginary parts of relative impedance are close to 1 and 0 , respectively, which means the impedance of the absorber matches well with the free space, resulting in a broad absorption bandwidth.

$$
\begin{gathered}
\chi_{e s}=\frac{2 i}{k_{0}} \frac{1-S_{11}}{1+S_{11}} \\
\chi_{m s}=\frac{2 i}{k_{0}} \frac{1+S_{11}}{1-S_{11}} \\
\varepsilon_{e f f}=1+\frac{\chi_{e s}}{d} \\
\mu_{e f f}=1+\frac{\chi_{m s}}{d} \\
Z_{r}=\sqrt{\frac{\mu_{e f f}}{\varepsilon_{e f f}}}
\end{gathered}
$$

Table 3. Calculations of relative impedance of the proposed structure at peak frequencies.

\begin{tabular}{ccc}
\hline Frequency $\mathbf{( T H z )}$ & Real Part of $\mathbf{Z r}$ & Imaginary Part of $\mathbf{Z r}$ \\
\hline Peak I 4.49 & 1.027 & -0.01 \\
Peak II 6.73 & 1.021 & -0.1673 \\
Peak III 9.66 & 0.9495 & -0.02 \\
\hline
\end{tabular}




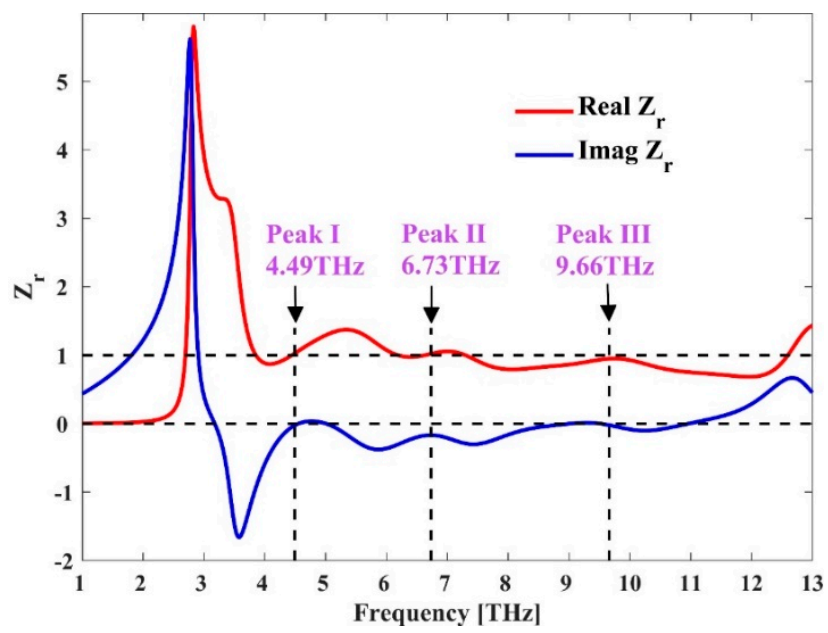

Figure 6. Real and imaginary parts of the relative impedance $Z_{r}$.

In order to illustrate the working principle more intuitively, the electric field distributions on the $\mathrm{x}-\mathrm{y}$ plane at frequencies of $4.49,6.73$, and $9.66 \mathrm{THz}$ corresponding to absorption peaks I-III for TE polarization are shown in Figure 7. Figure 7a-c show the two-dimensional electric-field profiles of the graphene layer at the three frequencies, while Figure $7 \mathrm{~d}-\mathrm{f}$ present the two-dimensional electric field distribution of the BDS layer. At $4.49 \mathrm{THz}$, the electric field distribution of the graphene layer is mainly concentrated in the narrow gaps along the $x$ and $y$ directions and the internal oblique gaps, while the electric field distribution of the BDS layer is mainly concentrated in the wide channels along the $x$ direction. At $6.73 \mathrm{THz}$, the electric field of the graphene layer is concentrated in each slit except for the four internal oblique slits, while the electric field of the BDS layer is scattered in the square blank channels. At $9.66 \mathrm{THz}$, the electric field of the graphene layer is concentrated in each slit except for the four slits in the $x$ direction, and the electric field of the BDS layer is concentrated on the four sides of the $x$ direction of the outer frame.

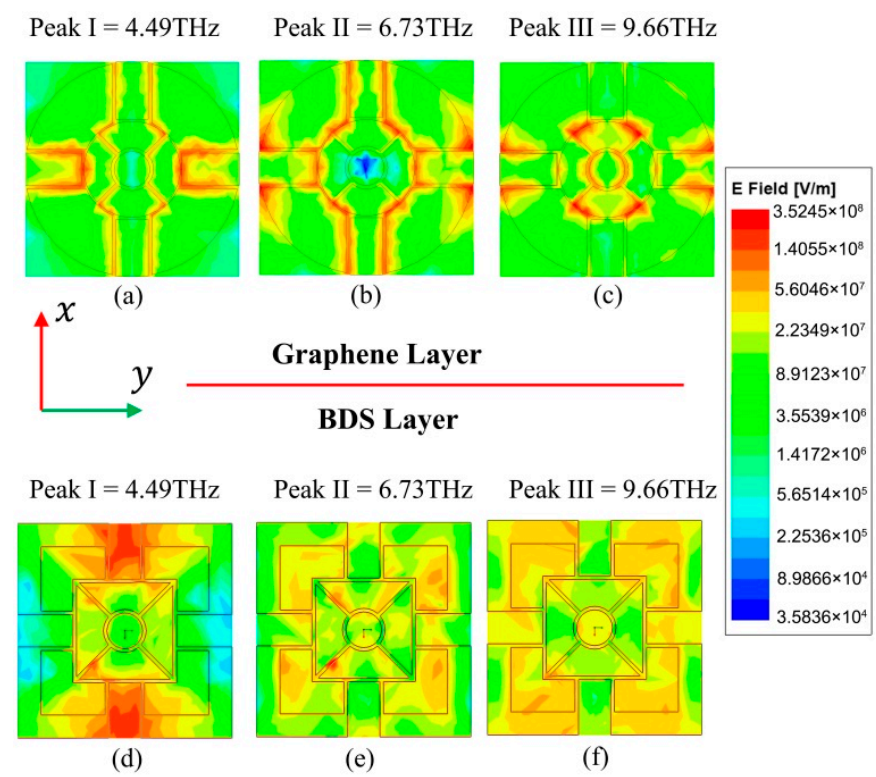

Figure 7. Absolute electric field distributions of graphene at (a) $4.49 \mathrm{THz}$, (b) $6.73 \mathrm{THz}$, and (c) $9.66 \mathrm{THz}$. Absolute electric field distributions of BDS at (d) $4.49 \mathrm{THz},(\mathbf{e}) 6.73 \mathrm{THz}$, and (f) $9.66 \mathrm{THz}$.

On the whole, as the frequency increases, the intensity of the electric field first increases and then decreases as the frequency increases in the graphene layer. As for the BDS layer, the change trend is opposite and firstly decreases and then increases. This is because that the incident wave excites carriers 
to oscillate along the $x$-axis and induce tangential electric fields on both graphene and BDS layers, which will cause energy loss. The energy consumption inside the loss materials including graphene, BDS and $\mathrm{Al}_{2} \mathrm{O}_{3}$ can be calculated by:

$$
A(f)=2 \pi f \varepsilon^{\prime \prime} \int_{v}\left|E_{l}\right|^{2} d V
$$

where $\varepsilon^{\prime \prime}$ is the imaginary part of dielectric constant, $V$ is the volume of lossy material, and $E_{l}$ is the electric field inside the lossy materials. In the range of 1-13 THz, the imaginary parts of graphene and BDS are large. Therefore, the electromagnetic energy of $\mathrm{THz}$ wave will be dissipated where the electric field is strong.

In order to understand how the energy is located at the resonance frequency, the electric field distribution in the $\mathrm{y}-\mathrm{z}$ plane is simulated to reveal where absorption mainly occurs. Figure 8a-c show the cross-sectional views of the electric field $|\mathrm{E}|$. It can be seen that the electric field is not only concentrated in different parts of the graphene and BDS patterns, but also trapped inside the dielectric layer, which means that the graphene pattern and BDS pattern, as well as the $\mathrm{Al}_{2} \mathrm{O}_{3}$ layer play an important role in absorption. The absorption contribution rate of each part is shown in Figure 9. We can see that the absorption in the graphene and BDS patterns is stronger than that in the $\mathrm{Al}_{2} \mathrm{O}_{3}$ layer, which is consistent with the absolute electric field distributions in Figures 7 and 8 . It could be mainly attributed to that the imaginary part of the dielectric constant of graphene and BDS is larger than that of the $\mathrm{Al}_{2} \mathrm{O}_{3}$ layer in the $\mathrm{THz}$ frequency range. Due to the symmetry of the structure, the TM polarization is the same as the TE polarization.

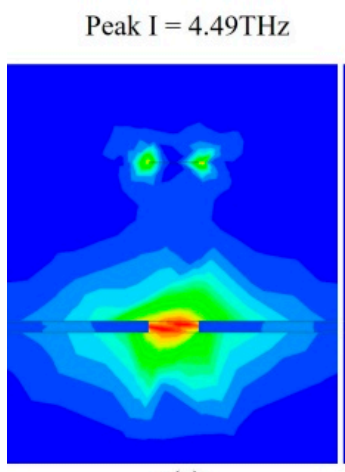

(a)

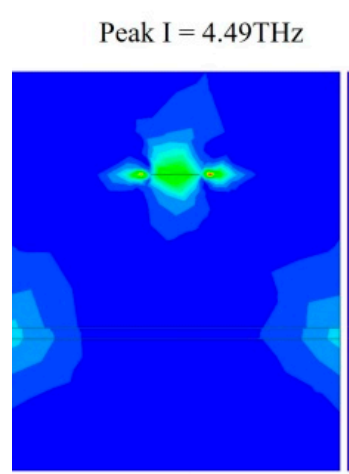

(d)

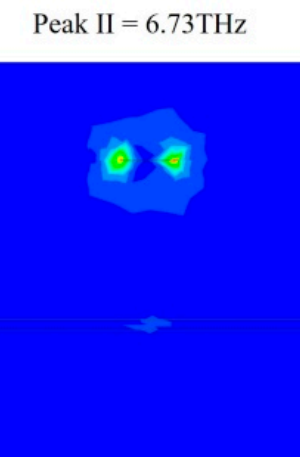

(b)

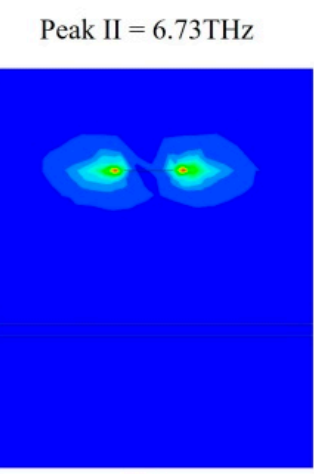

(e)
Peak III $=9.66 \mathrm{THz}$

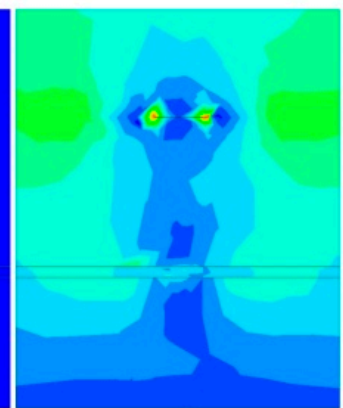

(c)

Peak III $=9.66 \mathrm{THz}$

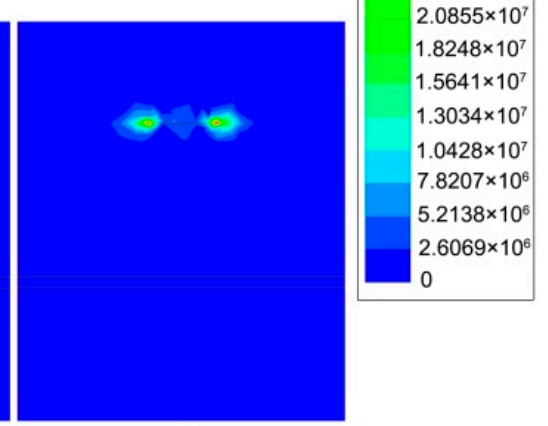

(f)

Figure 8. Absolute electric field distributions in the $y-z$ planes to illustrate the energy localization at $\mathrm{f}=4.49 \mathrm{THz}, 6.73 \mathrm{THz}$, and $9.66 \mathrm{THz}$. $(\mathbf{a}-\mathbf{c})$ and $(\mathbf{d}-\mathbf{f})$ are the two adjacent faces in the structure. 


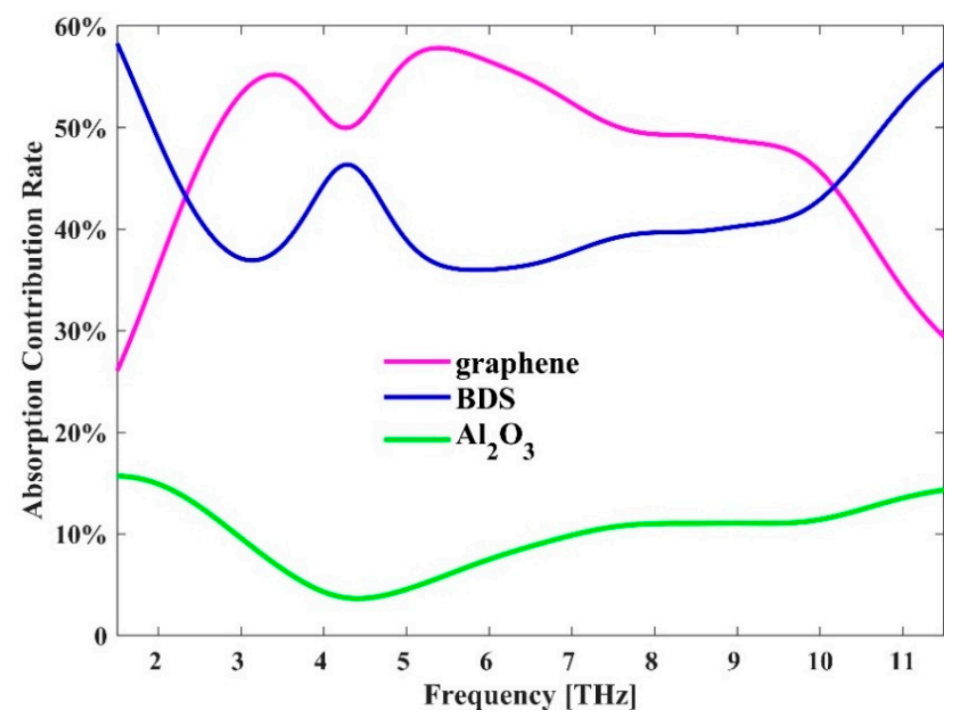

Figure 9. The absorption contribution rate of graphene, $\mathrm{BDS}$ and $\mathrm{Al}_{2} \mathrm{O}_{3}$ in the proposed hybrid absorber.

\section{Conclusions}

In summary, a tunable hybrid graphene-BDS broadband THz absorber is designed and studied. Due to the combination of advantages of graphene and BDS, the structure can achieve a high absorption exceeding $90 \%$ over a broad wavelength range of $6.64 \mathrm{THz}$. By adjusting the Fermi energy level of graphene and BDS, the absorption peaks and bandwidth can be dynamically tuned without reconstructing the structure. Besides, the absorber is insensitive to incident angles, yielding a high absorption over $90 \%$ at a large incident angle of $60^{\circ}$ and $70^{\circ}$ for TE and TM modes, respectively. Owing to its excellent performance, the proposed absorber has great potential in miniaturized ultra-broadband $\mathrm{THz}$ devices and microsystems.

Author Contributions: Conceptualization, J.W., Y.Z. and X.Y. (Xin Yan); formal analysis, J.W., X.Y. (Xin Yan) and X.Y. (Xueguang Yuan); methodology, J.W. and X.Y. (Xin Yan); resources, J.W., X.Z.; software, J.W.; writing-original draft preparation, J.W.; writing-review and editing, J.W., X.Y. (Xin Yan) and X.Z. All authors have read and agreed to the published version of the manuscript.

Funding: This work was supported by the National Natural Science Foundation of China (61935003, 61774021, and 61911530133), the National Key Research and Development Program of China (2018YFB2200104), Beijing Municipal Science and Technology Commission (Z191100004819012), the Fundamental Research Funds for the Central Universities (2018XKJC05), and the Fund of State Key Laboratory of Information Photonics and Optical Communications (Beijing University of Posts and Telecommunications), P. R. China (IPOC2020ZZ01 and IPOC2019ZT07).

Conflicts of Interest: The authors declare no conflict of interest.

\section{References}

1. Federici, J.; Moeller, L. Review of terahertz and subterahertz wireless communications. J. Appl. Phys. 2010, 107, 111101. [CrossRef]

2. Guo, C.; Zhang, J.; Xu, W.; Liu, K.; Yuan, X.; Qin, S.; Zhu, Z. Graphene-based perfect absorption structures in the visible to terahertz band and their optoelectronics applications. Nanomaterials 2018, 8, 1033. [CrossRef] [PubMed]

3. Jepsen, P.U.; Cooke, D.G.; Koch, M. Terahertz spectroscopy and imaging-Modern techniques and applications. Laser Photonics Rev. 2011, 5, 125-159. [CrossRef]

4. Xu, Z.; Wu, D.; Liu, Y.; Liu, C.; Yu, Z.; Yu, L.; Ye, H. Design of a tunable ultra-broadband terahertz absorber based on multiple layers of graphene ribbons. Nanoscale Res. Lett. 2018, 13, 143. [CrossRef] [PubMed]

5. Zhang, Y.; Feng, Y.; Zhu, B.; Zhao, J.; Jiang, T. Graphene based tunable metamaterial absorber and polarization modulation in terahertz frequency. Opt. Express 2014, 22, 22743-22752. [CrossRef] [PubMed] 
6. Xiong, H.; Yang, F. Ultra-broadband and tunable saline water-based absorber in microwave regime. Opt. Express 2020, 28, 5306-5316. [CrossRef] [PubMed]

7. Geim, A.K. Graphene: Status and prospects. Science 2009, 324, 1530-1534. [CrossRef] [PubMed]

8. Xiang, J.; Hu, S.; Lyu, M.; Zhu, W.; Ma, C.; Chen, Z.; Steglich, F.; Chen, G.; Sun, P. Large transverse thermoelectric figure of merit in a topological Dirac semimetal. Sci. China Phys. Mech. 2019, 63, 237011. [CrossRef]

9. Wieder, B.J.; Wang, Z.; Cano, J.; Dai, X.; Schoop, L.M.; Bradlyn, B.; Bernevig, B.A. Strong and fragile topological Dirac semimetals with higher-order Fermi arcs. Nat. Commun. 2020, 11, 627. [CrossRef]

10. Jiang, Y.; Wan, X.; Wang, J.; Wang, J. Tunable terahertz absorber based on bulk-Dirac-semimetal metasurface. IEEE Photonics J. 2018, 10, 4600607. [CrossRef]

11. Xiong, H.; Shen, Q.; Ji, Q. Broadband dynamically tunable terahertz absorber based on a Dirac semimetal. Appl. Opt. 2020, 59, 4970-4976. [CrossRef] [PubMed]

12. Wu, P.; Chen, Z.; Zhang, C.; Jian, R. A narrow dual-band monolayer unpatterned graphene-based perfect absorber with critical coupling in the near infrared. Micromachines 2020, 11, 58. [CrossRef] [PubMed]

13. Raad, S.H.; Atlasbaf, Z. Broadband continuous/discrete spectrum optical absorber using graphene-wrapped fractal oligomers. Opt. Express 2020, 28, 18049-18058. [CrossRef] [PubMed]

14. Yi, Z.; Chen, J.; Cen, C.; Chen, X.; Zhou, Z.; Tang, Y.; Ye, X.; Xiao, S.; Luo, W.; Wu, P. Tunable graphene-based plasmonic perfect metamaterial absorber in the THz region. Micromachines 2019, 10, 194. [CrossRef]

15. Chen, M.; Chen, C.; Deng, S.; Liu, H.; Teng, C.; Cheng, Y.; Yang, H.; Xu, R.; Deng, H.; Yuan, L. Dynamically tunable polarization-independent terahertz absorber based on bulk Dirac semimetals. OSA Contin. 2019, 2, 2477-2486. [CrossRef]

16. Meng, H.; Shang, X.; Xue, X.; Tang, K.; Xia, X.; Zhai, X.; Liu, Z.; Chen, J.; Li, H.; Wang, L. Bidirectional and dynamically tunable THz absorber with Dirac semimetal. Opt. Express 2019, 27, 31062-31074. [CrossRef]

17. Fang, P.; Shi, X.; Liu, C.; Zhai, X.; Li, H.; Wang, L. Single- and dual-band convertible terahertz absorber based on bulk Dirac semimetal. Opt. Commun. 2020, 462, 125333. [CrossRef]

18. Li, Y.; Zhai, X.; Xia, S.; Li, H.; Wang, L. Active control of narrowband total absorption based on terahertz hybrid Dirac semimetal-graphene metamaterials. J. Phys. D Appl. Phys. 2020, 53, 205106. [CrossRef]

19. Hanson, G.W. Dyadic Green's functions and guided surface waves for a surface conductivity model of graphene. J. Appl. Phys. 2008, 103, 064302. [CrossRef]

20. Bolotin, K.I.; Sikes, K.J.; Jiang, Z.; Klima, M.; Fudenberg, G.; Hone, J.; Kim, P.; Stormer, H.L. Ultrahigh electron mobility in suspended graphene. Solid State Commun. 2008, 146, 351-355. [CrossRef]

21. Kotov, O.V.; Lozovik, Y.E. Dielectric response and novel electromagnetic modes in three-dimensional Dirac semimetal films. Phys. Rev. B 2016, 93, 235417. [CrossRef]

22. Wikipedia. Available online: https://en.wikipedia.org/wiki/Riemann $\%$ E2 $\% 80 \% 93$ Siegel_theta_function (accessed on 20 August 2020).

23. Fu, P.; Liu, F.; Ren, G.J.; Su, F.; Li, D.; Yao, J.Q. A broadband metamaterial absorber based on multi-layer graphene in the terahertz region. Opt. Commun. 2018, 417, 62-66. [CrossRef]

24. Wang, T.; Zhang, Y.; Zhang, H.; Cao, M. Dual-controlled switchable broadband terahertz absorber based on a graphene-vanadium dioxide metamaterial. Opt. Mater. Express 2020, 10, 369-386. [CrossRef]

Publisher's Note: MDPI stays neutral with regard to jurisdictional claims in published maps and institutional affiliations.

(C) 2020 by the authors. Licensee MDPI, Basel, Switzerland. This article is an open access article distributed under the terms and conditions of the Creative Commons Attribution (CC BY) license (http://creativecommons.org/licenses/by/4.0/). 